\title{
ABSOLUTELY CONTINUOUS INVARIANT MEASURES FOR A CLASS OF AFFINE INTERVAL EXCHANGE MAPS
}

\author{
ZAQUEU COELHO, ARTUR LOPES, AND LUIZ FERNANDO DA ROCHA
}

(Communicated by Linda Keen)

\begin{abstract}
We consider a class $\mathscr{A}$ of affine interval exchange maps of the interval and we analyse several ergodic properties of the elements of this class, among them the existence of absolutely continuous invariant probability measures. The maps of this class are parametrised by two values $a$ and $b$, where $a, b \in(0,1)$. There is a renormalization map $T$ defined from $\mathscr{A}$ to itself producing an attractor given by the set $\mathscr{R}$ of pure rotations, i.e. the set of $(a, b)$ such that $b=1-a$. The density of the absolutely continuous invariant probability and the rotation number of the elements of the class $\mathscr{A}$ are explicitly calculated. We also show how the continued fraction expansion of this rotation number can be obtained from the renormalization map.
\end{abstract}

\section{INTRODUCTION}

Let $a, b \in(0,1)$ and consider the map $f:[0,1) \rightarrow[0,1)$ given by

$$
f(x)= \begin{cases}a+\frac{1-a}{b} x, & \text { for } 0 \leq x<b ; \\ \frac{a}{1-b}(x-b), & \text { for } b \leq x<1 .\end{cases}
$$

The graph of $f$ is shown in Figure 1 on the next page. Let $\mathscr{A}^{*}$ denote the class of such maps. We show in the first part of this paper that every map in $\mathscr{A}^{*}$ has an absolutely continuous invariant probability measure. We also obtain a formula for the density of the invariant measure and the rotation number of the map in terms of the parameters $a$ and $b$. In the second part of the paper we define a renormalization map acting on $\mathscr{A}^{*}$ and we show that the set of pure rotations is an attractor for that map. In addition we show how the continued fraction expansion of the rotation number of a map $f \in \mathscr{A}^{*}$ can be obtained from the renormalization map.

We should note that in the case the rotation number of $f \in \mathscr{A}^{*}$ is irrational, the existence of an absolutely continuous invariant probability measure for $f$

Received by the editors January 26, 1994.

1991 Mathematics Subject Classification. Primary 58F11, 58F03.

Key words and phrases. Affine interval mappings, invariant measures, absolute continuity.

This work is part of Projeto Temático de Equipe "Transição de Fase Dinâmica em Sistemas Evolutivos" financially supported by FAPESP grant 90/3918-5. During part of this work, the first named author received financial support by FAPESP grant 93/4129-2. 


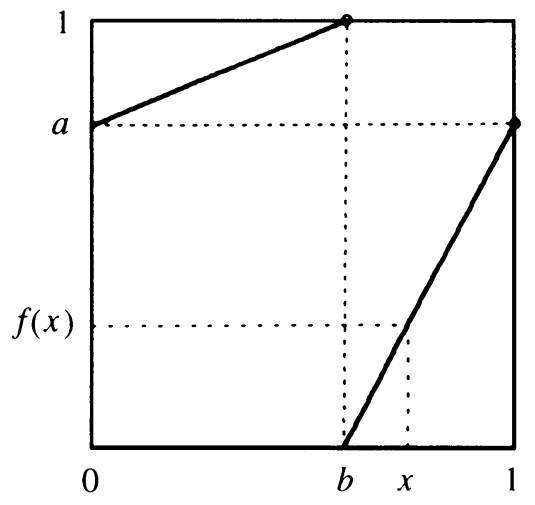

FIGURE 1

can be proved using the idea contained in the proof of Theorem 2 below and Proposition VI.7.7.1 of [He], the latter being a result by Petersen [Pe]. Here we give a different (also simple) proof of this fact which enables us to compute explicitly the density of the invariant measure and the rotation number of the map. In relation to this problem we should point out that in Section VI.7 of [He], Herman considers a class of piecewise affine homeomorphisms of the circle (with two slopes) which does not admit invariant $\sigma$-finite measures.

After completing an earlier version of this paper, we learned that Boshernitzan [Bo] has previously considered the class of maps $\mathscr{A}^{*}$ to give an example of a homeomorphism of the circle with the property that the restriction of the map to the set of rationals $\mathbb{Q} \subseteq[0,1)$ is a bijection onto $\mathbb{Q}$, and the orbit of every rational is dense in the circle. The example is produced by taking rational parameters $a$ and $b$ such that the rotation number of the map is irrational. In the existence proof of this result, an explicit expression for the density of the invariant measure and the rotation number in terms of the slopes of the map is mentioned.

Now we state some conventions we will apply throughout this paper. Since the values of $a=f(0)$ and $b=f^{-1}(0)$ uniquely define the map $f$, the set $\mathscr{A}^{*}$ can be parametrised by the open square $(0,1) \times(0,1)$ and we will often refer to $f$ by the pair of parameters $(a, b)$. The parameter $b$ is referred to as the critical point of $f$. We will denote the derivative $D f$ by $\alpha=(1-a) / b$ on $[0, b)$, and by $\beta=a /(1-b)$ on $[b, 1)$.

The map $f$ induces a piecewise affine homeomorphism of the circle which we also denote by $f$. Since the derivative of $f$ has bounded variation, recall from the Denjoy Theorem that if the rotation number $\rho$ of $f$ is irrational, then there are no wandering intervals for $f$, and equivalently, $f$ is conjugate to the rotation by $\rho$ (cf. [De 1,2]). In the case $b=1-a$, then $f$ is the rotation by $a$. We shall denote the latter subset of $\mathscr{A}^{*}$ by $\mathscr{R}^{*}$ and refer to it as the set of pure rotations. The symbols $\mathscr{A}$ and $\mathscr{R}$ are reserved to denote $\mathscr{A}^{*} \cup\{$ Id $\}$ and $\mathscr{R}^{*} \cup\{$ Id $\}$ respectively, where Id stands for the identity map on $[0,1)$.

\section{The INVARIANT MEASURE}

In order to show that every $f \in \mathscr{A}^{*}$ has an invariant probability which is equivalent to Lebesgue measure, we need the following results. 
Proposition 1. Let $f \in \mathscr{A}^{*}$ be given. Then either there exists $m>1$ such that $f^{m}=$ Id or $f^{n} \in \mathscr{A}^{*}$ for every $n \geq 1$. In the latter case, let the pair $\left(a_{n}, b_{n}\right)$ represent $f^{n}$ and let $\alpha_{n}$ and $\beta_{n}$ be the derivatives of $f^{n}$ on $\left[0, b_{n}\right)$ and $\left[b_{n}, 1\right)$ respectively. Then for all $n \geq 1$ we have

$$
\frac{\beta_{n}}{\alpha_{n}}=\frac{\beta}{\alpha} .
$$

Proof. Assume that $f^{n} \in \mathscr{A}^{*}$ and that (1) holds. We will show that either $f^{n+1}=$ Id , or $f^{n+1} \in \mathscr{A}^{*}$ and (1) holds for $n+1$.

Recall from the definition of the parameters of $f^{n}$ that $a_{n}=f^{n}(0)$ and $b_{n}=f^{-n}(0)$. Define $z=f^{-1}\left(b_{n}\right)$; then we note that $f^{n+1}(z)=0$. Now the only points where $f^{n+1}$ could be discontinuous are $b$ and $z$. However, $f^{n+1}$ is continuous at $b$ since

$$
\lim _{x \rightarrow b_{-}} f^{n+1}(x)=\lim _{y \rightarrow 1_{-}} f^{n}(y)=a_{n}
$$

and

$$
\lim _{x \rightarrow b_{+}} f^{n+1}(x)=\lim _{y \rightarrow 0_{+}} f^{n}(y)=a_{n} .
$$

Next we show that $f^{n+1}$ is linear on each of the intervals $[0, z)$ and $(z, 1]$ if $z \neq 0$, and $f^{n+1}=$ Id if $z=0$. First suppose that $z \neq 0$ and $b>z$; then in this case the derivative $D f^{n+1}$ is constant by parts and equal to $\alpha \alpha_{n}, \alpha \beta_{n}$ and $\beta \alpha_{n}$, respectively, on $[0, z),[z, b)$ and $[b, 1)$. Since by hypothesis $\beta_{n} / \alpha_{n}=\beta / \alpha$, we conclude that the derivative $D f^{n+1}$ matches on both sides of $b$. Therefore $f^{n+1} \in \mathscr{A}^{*}, b_{n+1}=z, a_{n+1}=f^{n}(a), \alpha_{n+1}=\alpha \alpha_{n}$ and $\beta_{n+1}=\alpha \beta_{n}$. Consequently we have

$$
\frac{\beta_{n+1}}{\alpha_{n+1}}=\frac{\beta_{n}}{\alpha_{n}}=\frac{\beta}{\alpha} .
$$

We note from the above proof that in the case $z=0$ we have $f^{n+1}$ linear on $[0,1)$ and hence $f^{n+1}$ must be the identity map on $[0,1)$.

Now suppose $z \neq 0$ and $b<z$. In this case, the derivative $D f^{n+1}$ is constant by parts and equal to $\alpha \beta_{n}, \alpha_{n} \beta$ and $\beta_{n} \beta$, respectively, on $[0, b)$, $[b, z)$ and $[z, 1)$. Thus we also conclude that the derivative $D f^{n+1}$ matches on both sides of $b$. Therefore $f^{n+1} \in \mathscr{A}^{*}, b_{n+1}=z, a_{n+1}=f^{n}(a), \alpha_{n+1}=\alpha \beta_{n}$ and $\beta_{n+1}=\beta \beta_{n}$, which implies

$$
\frac{\beta_{n+1}}{\alpha_{n+1}}=\frac{\beta}{\alpha}
$$

Remark 1. From the above proposition and the fact that every $f \in \mathscr{A}^{*}$ does not have fixed points, we conclude that $f^{n} \in \mathscr{A}^{*}$ for all $n \geq 1$ if and only if the rotation number of $f$ is irrational. Alternatively, there exists $m>1$ such that $f^{m}=$ Id if and only if the rotation number of $f$ is rational.

Proposition 2. For each $f \in \mathscr{A}^{*}$ there exists $M \geq 1$ such that for all $n \geq 1$,

$$
1 / M \leq\left\|D f^{n}\right\| \leq M
$$

Proof. We show that $M=\max \{\alpha / \beta ; \beta / \alpha\}$ satisfies (2). Suppose $f$ is such that $f^{n} \in \mathscr{A}^{*}$ for all $n \geq 1$, and $\alpha<\beta$. Then $\alpha_{n}<1<\beta_{n}$ for all $n \geq 1$, and for $x \in\left[0, b_{n}\right)$ we have

$$
1 / M \leq \frac{\alpha}{\beta}<\beta_{n} \frac{\alpha}{\beta}=\alpha_{n}=\left|D f^{n}(x)\right|<1
$$


Also for $x \in\left[b_{n}, 1\right)$ we have

$$
1<\left|D f^{n}(x)\right|=\beta_{n}=\alpha_{n} \frac{\beta}{\alpha}<\frac{\beta}{\alpha} \leq M .
$$

If $\alpha>\beta$ a similar inequality holds. Now if $\alpha=\dot{\beta}$, then $f$ is a pure rotation and (2) also holds. Finally, in the case $f^{m}=$ Id for some $m>1$, where $m$ is the least possible with this property, the maximum of $\left|D f^{n}\right|$ is attained by $\left|D f^{j}\right|$ for some $1 \leq j<m$. Since these derivatives satisfy (1) we conclude from the above proof that (2) also holds in this case.

Theorem 1. There exists an absolutely continuous invariant probability for $f \in$ $\mathscr{A}^{*}$.

Proof. If $f$ has parameters $(a, b)$, then the inverse $f^{-1}$ has parameters $(b, a)$. Therefore, from Proposition 2, $D f^{n}$ and $D f^{-n}$ are uniformly bounded away from zero and infinity. Hence the function $\varphi(x)=\sup _{n \in \mathbf{Z}}\left|D f^{n}(x)\right|$ is well defined and satisfies $\varphi(f(x))|D f(x)|=\varphi(x)$. Consequently $d \mu(x)=\varphi(x) d x$ defines an absolutely continuous invariant measure for $f$. Note from Proposition 2 that $\mu$ is equivalent to Lebesgue measure. Normalising $\varphi$ by the $\mathrm{L}^{1}$-norm we obtain an invariant probability.

Now we derive a formula for the rotation number of $f \in \mathscr{A}^{*}$ in terms of its parameters.

Theorem 2. Let $f \in \mathscr{A}^{*}$ have parameters $(a, b)$ and derivatives $\alpha, \beta$ on $[0, b),[b, 1)$ respectively. Then the rotation number $\rho$ of $f$ is given by

$$
\rho(a, b)=\log \frac{1}{\alpha} / \log \frac{\beta}{\alpha}=\log \frac{b}{1-a} / \log \frac{a b}{(1-b)(1-a)} .
$$

Proof. Suppose $\rho$ is irrational. Then $f$ is uniquely ergodic and we let $\mu$ be its unique invariant probability. From Theorem 1 we conclude that $\mu$ is equivalent to Lebesgue measure and therefore there are no wandering intervals for $f$. Let $h$ be given by $h(x)=\mu[0, x)$. It is well known that $h$ is a conjugacy between $f$ and the pure rotation by $\rho$. Since $\rho=\mu[0, f(0))=\mu[0, a)$ and $\mu$ is $f$-invariant, we obtain $\rho=\mu[0, a)=\mu\left(f^{-1}[0, a)\right)=\mu[b, 1)$. Now from the Ergodic Theorem and Proposition 2 we have

$$
0=\lim _{n \rightarrow \infty} \frac{1}{n} \log \left|D f^{n}(x)\right|=\int \log |D f(x)| d \mu(x)
$$

which implies

$$
0=\mu[0, b) \log \alpha+\mu[b, 1] \log \beta=(1-\rho) \log \alpha+\rho \log \beta .
$$

The above equation gives (3) in the case $\rho$ is irrational. Finally, suppose $\rho=$ $p / q$ in its irreducible form. Then we know that there exists a periodic point $x_{0}$ with least period $q$ and there are no periodic points with least period strictly less than $q$. Therefore from Proposition 1 we deduce that $f^{q}=\operatorname{Id}$ and $f^{q-1} \in \mathscr{A}^{*}$. Now studying the orbit of any point $x_{0}$ we conclude that $p$ is the number of times this orbit visits the interval $[b, 1)$. Since every point is periodic of period $q$ and the derivative of $f^{q}$ is identically equal to 1 we obtain the relation $\alpha^{q-p} \beta^{p}=1$. Applying logarithms we conclude that equation (3) is also satisfied in this case. 
Theorem 3. Let $f \in \mathscr{A}^{*}$ have irrational rotation number. Then the density of the unique invariant probability for $f$ is given by

$$
\frac{1}{c_{\alpha, \beta}} \cdot \frac{\alpha}{\alpha x+\beta(1-x)},
$$

where the normalising constant $c_{\alpha, \beta}$ is given by $\frac{\alpha}{\beta-\alpha} \log \frac{\beta}{\alpha}$.

Proof. A direct computation shows that the above density satisfies the functional equation

$$
\varphi(x)=\varphi(f(x))|D f(x)|,
$$

and defines a probability measure. Therefore the result follows. However, we show how the expression was originally derived. We first note that for every $n \geq 1$ we have

$$
b_{n} \alpha_{n}+\left(1-b_{n}\right) \beta_{n}=1 \text {, }
$$

and therefore by Proposition 1 we obtain

$$
b_{n} \alpha_{n}+\left(1-b_{n}\right) \alpha_{n} \frac{\beta}{\alpha}=1 .
$$

Since the sequence $\left\{b_{n}\right\}$ is dense in $[0,1)$, there exists a subsequence $b_{n_{k}}$ converging to a given $x \in(0,1)$. Now from (5) the $\operatorname{limit} \lim _{k \rightarrow \infty} \alpha_{n_{k}}=\tilde{\alpha}$ exists and satisfies the equation

$$
x \tilde{\alpha}+(1-x) \tilde{\alpha} \frac{\beta}{\alpha}=1 .
$$

From the above we obtain

$$
\tilde{\alpha}=\frac{\alpha}{\alpha x+\beta(1-x)} .
$$

Using a simple geometric argument (the fact that the area under the graph of $D f^{n}(x)$ is one and that $\left.\alpha_{n}=\alpha \beta_{n} / \beta\right)$ we conclude that $\tilde{\alpha}=\sup _{n \in \mathbf{Z}}\left|D f^{n}(x)\right|$. As used in Theorem 2, this supremum is an invariant density and after normalising we obtain (4).

\section{THE RENORMALIZATION MAP}

Following Rauzy [Ra1,2], we define a renormalization map $T$ acting on $\mathscr{A}=$ $\mathscr{A}^{*} \cup\{\mathrm{Id}\}$ in the following way. Let $f \in \mathscr{A}^{*}$ have parameters $(a, b)$ and let $I$ be the largest of the intervals $[0, a)$ and $[0, b)$. Then the renormalization map $T(f):[0,1) \rightarrow[0,1)$ is obtained by inducing the map $f$ on $I$ and rescaling $I$ to the unit interval. If $a \neq b$, then $T(f) \in \mathscr{A}^{*}$ and as a map on the parameter space $Q=(0,1) \times(0,1), T(f)$ has parameters

$$
T(a, b)= \begin{cases}\left(\frac{a-b}{1-b}, \frac{b}{a}\right) & \text { if } b<a, \\ \left(\frac{a}{b}, \frac{b-a}{1-a}\right) & \text { if } a<b .\end{cases}
$$

If $a=b$, then $T(f)$ is the identity map on $[0,1)$. By identifying the points $(1,0)$ and $(0,1)$ to the identity map, and considering it to be a fixed point of the renormalization map, we obtain an extension of $T$ to $\mathscr{A} \cong Q \cup\{(1,0) \simeq$ 


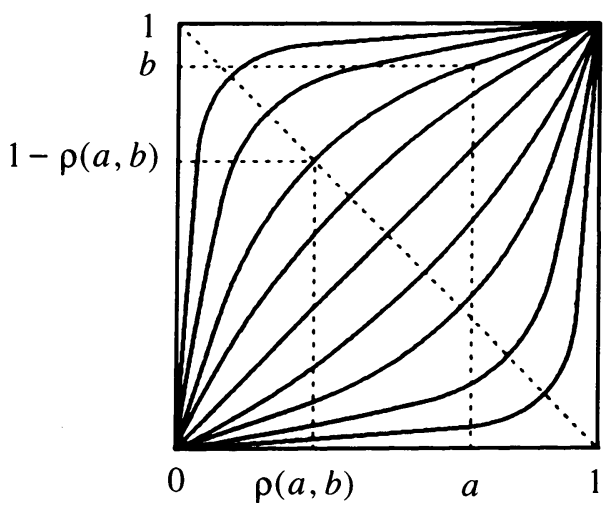

FIGURE 2

$(0,1)\}$. Putting a topology on $\mathscr{A}$ induced by the quotient topology on $Q \cup$ $\{(1,0) \simeq(0,1)\}$, we note that $T$ is continuous on $\mathscr{A}^{*}$ and discontinuous at the identity map.

Let $\mathscr{F}$ be the foliation of $\mathscr{A}^{*} \cong Q$ by the curves $\rho(a, b)=$ const, where $\rho(a, b)$ is given in (3) (see Figure 2). Including in $\mathscr{F}$ the singular leaf consisting of the identity map and associating it to the rotation number zero, $\mathscr{F}$ becomes a foliation of $\mathscr{A}$. We recall that $\mathscr{R}=\mathscr{R}^{*} \cup\{$ Id $\}$ is the set of pure rotations, where the identity map is regarded as pure rotation by angle zero.

Proposition 3. The foliation $\mathscr{F}$ is invariant under the renormalization map $T$, and the leaf through $f \simeq(a, b)$ intersects $\mathscr{R}$ in $(\rho, 1-\rho)$, where $\rho$ is the rotation number of $f$.

Proof. Let $\rho=\rho(a, b)$ and suppose $a>b$. From (3) we have

$$
\frac{b^{1-\rho}}{(1-a)^{1-\rho}}=\frac{a^{\rho}}{(1-b)^{\rho}} \text {. }
$$

Note that in this case $\rho>1 / 2$. Now let $s$ be the rotation number of $T(f) \simeq$ $T(a, b)$. Then using the expression of $T$ in (6) we obtain

$$
\frac{b}{a} /\left(1-\frac{a-b}{1-b}\right)=\left(\frac{(a-b) b}{(1-b) a} /\left(\left(1-\frac{b}{a}\right)\left(1-\frac{a-b}{1-b}\right)\right)\right)^{s},
$$

and after a simplification we get

$$
\frac{b^{1-s}}{(1-a)^{1-s}}=\frac{a}{1-b} .
$$

Comparing with (7) we conclude that $s=2-1 / \rho$. Therefore $T$ takes the leaf of $\mathscr{F}$ corresponding to the maps $f \in \mathscr{A}$ with rotation number $\rho \in(1 / 2,1)$ to the leaf with rotation number $2-1 / \rho$.

A similar computation for $a<b$, which is equivalent to $\rho=\rho(a, b) \in$ $(0,1 / 2)$, shows that the rotation number of $T(f)$ is given by $\rho /(1-\rho)$. Finally, if $a=b$ (i.e. $\rho(a, b)=1 / 2$ ) we have $T(f)=\mathrm{Id}$, which has rotation number zero and corresponds to the singular leaf of $\mathscr{F}$. 


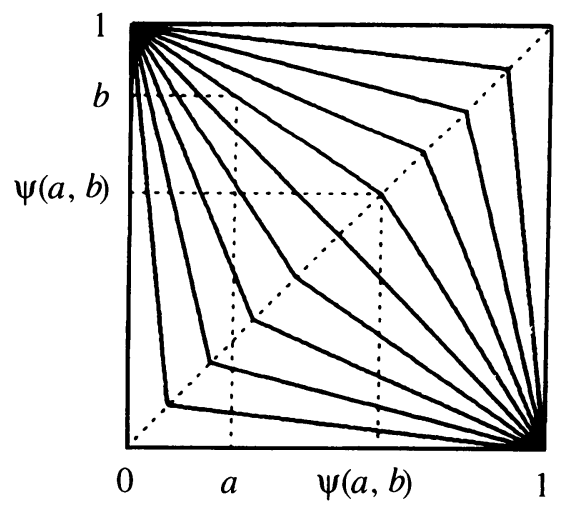

FIGURE 3

Now define a function $\psi: Q \cup\{(1,0) \simeq(0,1)\} \rightarrow(0,1)$ by

$$
\psi(a, b)=\left\{\begin{array}{cl}
\frac{a}{a+1-b}, & \text { if }(a, b) \in Q \text { and } a \leq b, \\
\frac{b}{b+1-a}, & \text { if }(a, b) \in Q \text { and } a \geq b, \\
1 / 2, & \text { if }(a, b)=(1,0) \simeq(0,1) .
\end{array}\right.
$$

We note that the function $\psi$ is continuous on $Q$ and discontinuous on $\{(1,0)$ $\simeq(0,1)\}$. The level curves $\psi=$ const define a continuous foliation $\mathscr{F}$ ' of $\mathscr{A}^{*}$ (see Figure 3), which is transverse to $\mathscr{F}$. We also note that $\mathscr{R}$ is the leaf of $\mathscr{F}^{\prime}$ corresponding to the level curve $\psi=1 / 2$. Moreover, the leaf corresponding to $\psi=s \in(0,1)$ intersects the diagonal $\{a=b\}$ at the point $(s, s)$.

Theorem 4. The set of pure rotations $\mathscr{R}$ is an attractor for the renormalization map $T$.

Proof. If we show that

$$
|\psi(T(a, b))-1 / 2|<|\psi(a, b)-1 / 2|,
$$

for all $b \neq 1-a$, then it means that the renormalization map $T$ takes a point in a leaf of $\mathscr{F}^{\prime}$ to another leaf of $\mathscr{F}^{\prime}$ which is strictly closer to $\mathscr{R}$. Since the only $T$-invariant leaf of $\mathscr{F}^{\prime}$ is $\mathscr{R}$, the result follows. Hence, it remains to prove $(8)$.

If $a=b$, then $\psi(a, a)=a$ and $T(a, a)=\mathrm{Id}$, therefore (8) is satisfied in this case since $\psi(\mathrm{Id})=1 / 2$. The open unit square $Q$ with the restrictions $b \neq 1-a$ and $a \neq b$ is divided into four triangles. Since the function $\varphi(a, b)=$ $|\psi(a, b)-1 / 2|$ satisfies $\varphi(1-a, 1-b)=\varphi(a, b)$ and $\varphi(T(1-a, 1-b))=$ $\varphi(T(a, b))$, for all $(a, b) \in Q$, we need only to check (8) on the interior of the semi-open triangles $D_{1}=\{(a, b): a \leq b, b>1-a\}$ and $D_{2}=\{(a, b): a \geq$ $b, b>1-a\}$.

The function $\varphi$ on $D_{1} \cup D_{2}$ is given by

$$
2 \varphi(a, b)= \begin{cases}\frac{a-(1-b)}{a+(1-b)}, & \text { if }(a, b) \in D_{1}, \\ \frac{b-(1-a)}{b+(1-a)}, & \text { if }(a, b) \in D_{2} .\end{cases}
$$




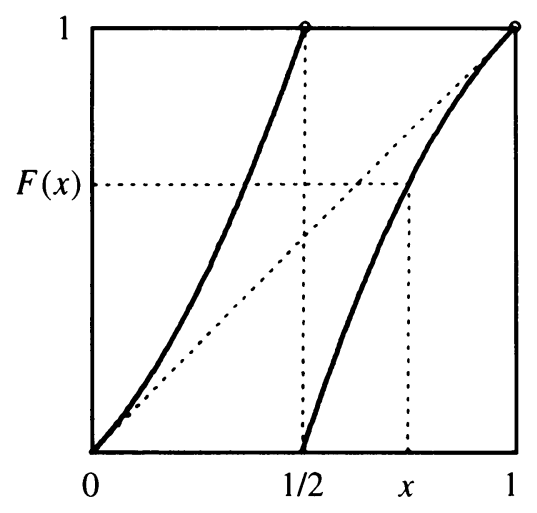

Figure 4

We note that $D_{1} \cup D_{2} \cup\{\mathrm{Id}\}$ is invariant under $T$. Therefore it remains to check (8) for $(a, b)$ in any of the sets of the form $D_{i} \cap T^{-1} D_{j}$ with $i, j=1,2$.

Suppose $(a, b) \in D_{1} \cap T^{-1} D_{1}$; then

$$
2 \varphi(T(a, b))=\frac{\frac{a}{b}-\left(1-\frac{b-a}{1-a}\right)}{\frac{a}{b}+\left(1-\frac{b-a}{1-a}\right)}=\frac{a\left(\frac{1-a}{b}\right)-(1-b)}{a\left(\frac{1-a}{b}\right)+(1-b)},
$$

and since $1-a<b$ we conclude that $\varphi(T(a, b))<\varphi(a, b)$ in this case. Now suppose $(a, b) \in D_{1} \cap T^{-1} D_{2} ;$ then

$$
2 \varphi(T(a, b))=\frac{\left(\frac{b-a}{1-a}\right)-\left(1-\frac{a}{b}\right)}{\left(\frac{b-a}{1-a}\right)+\left(1-\frac{a}{b}\right)}=\frac{b-(1-a)}{b+(1-a)}<\frac{a-(1-b)}{a+(1-b)},
$$

for all $(a, b) \in D_{1}$. Therefore, in this case, we also have $\varphi(T(a, b))<\varphi(a, b)$. The cases $(a, b) \in D_{2} \cap T^{-1} D_{1}$ and $(a, b) \in D_{2} \cap T^{-1} D_{2}$ can be treated in a similar way. This finishes the proof of $(8)$ and the theorem.

In order to understand the dynamics of $T$ around the attractor $\mathscr{R}$, we will need to recall some properties of the so-called backward continued fraction map of the interval. This is the map $F:[0,1) \rightarrow[0,1)$ defined by

$$
F(\rho)= \begin{cases}\frac{\rho}{1-\rho}, & \text { for } 0 \leq \rho<\frac{1}{2}, \\ 2-\frac{1}{\rho}, & \text { for } \frac{1}{2} \leq \rho<1 .\end{cases}
$$

The graph of this map is shown in Figure 4. We note that $F$ induces a differentiable map of the circle, with one indifferent fixed point at the origin (i.e. $D F(0)=1)$. This map admits an invariant measure with infinite mass and with density $1 /(x(1-x))$. The relation between $F$ and the renormalization map $T$ is expressed by the equation $T(\rho, 1-\rho)=(F(\rho), 1-F(\rho))$. Therefore the dynamics of $T$ on the attractor $\mathscr{R}$ is conjugate to $F$ acting on the unit interval. 
Consider the partition of the interval $[0,1)$ given by $A=[0,1 / 2)$ and $B=[1 / 2,1)$. For each $x \in[0,1)$ define a string of non-negative integers $c_{i}=$ $c_{i}(x)$ in the following way. If $x \in A$, then let $c_{1}$ be the first time the forward orbit of $x$ under $F$ leaves $A$, i.e. $F^{j}(x) \in A$ for $j \in\left\{0,1, \cdots, c_{1}-1\right\}$ and $F^{c_{1}}(x) \notin A$. Let $c_{2}$ be the number of times the forward orbit of $F^{c_{1}}(x)$ stays in $B$ before it returns to $A$, i.e. $F^{c_{1}+j}(x) \in B$ for $j \in\left\{0,1, \cdots, c_{2}-1\right\}$ and $F^{c_{2}}(x) \notin B$. Inductively define $c_{n}$ as the number of times the forward orbit of $F^{c_{n}}(x)$ stays in the same atom of the partition before visiting the other atom. In the case $x \in B$ define $c_{1}=0$, and let $c_{2}, c_{3}, \cdots$ be defined inductively as before. If one of the integers $c_{i}$ is infinite, then stop the sequence.

We make the convention that $1 / \infty=0$ in what follows. Using the fact that for every $n>1$ we have $F(1 / n)=1 /(n-1)$ and $F(n /(n+1))=(n-1) / n$, it is not difficult to prove that the continued fraction expansion of every number $x \in[0,1)$ is given by

$$
x=\frac{1}{\left(c_{1}+1\right)+\frac{1}{c_{2}+\frac{1}{c_{3}+\cdots}}}
$$

Let $d$ denote a distance on $[0,1)$ compatible with the circle identification. From the dynamics of $F$ on the continued fraction expansion of a number we note that if $x_{1}, x_{2} \in[0,1)$ are irrational numbers, then $d\left(F^{n}\left(x_{1}\right), F^{n}\left(x_{2}\right)\right)$ converges to zero as $n \rightarrow \infty$ if and only if there exists $N \geq 1$ such that the continued fraction expansion of $F^{N}\left(x_{1}\right)$ and $F^{N}\left(x_{2}\right)$ coincides. This is equivalent to the existence of $k \geq 1$ such that $c_{i}\left(x_{1}\right)=c_{i}\left(x_{2}\right)$ for all $i \geq k$ and $\sum_{j=1}^{k} c_{i}\left(x_{1}\right)=\sum_{j=1}^{k} c_{i}\left(x_{2}\right)$. We define an equivalence relation on $[0,1)$ by setting $x_{1} \sim x_{2}$ if there exists $k \geq 1$ satisfying the latter property.

Now let $\Delta^{+}$denote the open upper triangle $\{(a, b) \in Q: a<b\}$ and let $\Delta^{-}$be the semi-open lower triangle $\{(a, b) \in Q: a \geq b\}$. Replacing the map $F$ by the renormalization map $T$ in the above definition of the sequence $c_{i}$ and taking $A=\Delta^{+} \cup\{\mathrm{Id}\}$ and $B=\Delta^{-}$as a partition of $\mathscr{A}$, we obtain for each $f \in \mathscr{A}$ a sequence $c_{i}=c_{i}(f), i \geq 1$, corresponding to the successive number of times the orbit of $f$ under $T$ stays in $A$ or $B$. Using Proposition 3 we realise that the rotation number $\rho$ of $f$ has continued fraction expansion given by (9), where $c_{i}=c_{i}(f)$. This shows that the renormalization map can be used to determine the continued fraction expansion of the rotation number of any member of the class $\mathscr{A}$. The equivalence relation on $[0,1)$ defined above induces an equivalence relation on the leaves of the foliation $\mathscr{F}$ in the following way. Two leaves $L_{i}=\left\{f \in \mathscr{A}: \rho(f)=\rho_{i}\right\}, i=1,2$, are said to be equivalent if $\rho_{1} \sim \rho_{2}$. This is needed in our final result.

Theorem 5. The foliation $\mathscr{F}$ under the above equivalence is the stable manifold foliation of the attractor $\mathscr{R}$.

Proof. Let $R_{\alpha}$ be the pure rotation by $\alpha$. Suppose $f \in \mathscr{A}$ has rotation number $\rho$ and satisfies

$$
\lim _{n \rightarrow \infty} d_{\infty}\left(T^{n}\left(R_{\alpha}\right), T^{n}(f)\right)=0,
$$

where $d_{\infty}$ denotes the supremum distance. Since $T$ preserves the foliation 
$\mathscr{F}$, and $\mathscr{R}$ is an attractor for $T$, we also have

$$
\lim _{n \rightarrow \infty} d_{\infty}\left(T^{n}(f), T^{n}\left(R_{\rho}\right)\right)=0 .
$$

Hence, from the fact that $T$ restricted to $\mathscr{R}$ is conjugate to $F$ acting on $[0,1)$, we conclude that $d\left(F^{n}(\rho), F^{n}(\alpha)\right)$ converges to zero as $n \rightarrow \infty$. This shows that $\rho \sim \alpha$.

\section{REFERENCES}

[Bo] M. Boshernitzan, Dense orbits of rationals, Proc. Amer. Math. Soc. 117 (1993), 1201-1203.

[De1] A. Denjoy, Sur les courbes définies par les équations différentielles à la surface du tore, J. Math. Pures Appl. (9) 11 (1932), 333-375.

[De2] —_, Les trajectories à la surface du tore, C. R. Acad. Sci. Paris 223 (1946), 5-7.

[He] M. Herman, Sur la conjugaison differentiable des difféomorphismes du cercle a des rotations, Inst. Hautes Études Sci. Publ. Math. 49 (1979), 5-234.

[Pe] K. Petersen, On a series of cosecants related to a problem in ergodic theory, Compositio Math. 26 (1973), 313-317.

[Ra1] G. Rauzy, Une généralisation du developpment en fraction continue, Séminaire de Théorie des Nombres-Année, Paris.

[Ra2] _ Echanges d'intervalles et transformations induites, Acta Arith. 34 (1979), 315-328.

(Z. Coelho) Instituto de Matemática e Estatística, Universidade de São Paulo, CaiXa Postal 20570, CEP 01425-990, São PaUlo - SP, BRazIL

Current address, Z. Coelho: Mathematics Research Centre, University of Warwick, Coventry, CV4 7AL, United Kingdom

E-mail address: zaqueu@ime.usp.br

(A. Lopes and L. F. da Rocha) Instituto de Matemática, Universidade Federal do Rio Grande do Sul, Av. Bento Gonçalves, 9500, CEP 91500, Porto Alegre - RS, Brazil

$E$-mail address, A. Lopes: alopes@if 1 .ufrgs.br

E-mail address, L. F. da Rocha: Irocha@if1.ufrgs.br 